Check for updates

Cite this: Phys. Chem. Chem. Phys. 2022, 24, 4809

Received 27th August 2021,

Accepted 1st February 2022

DOI: 10.1039/d1cp03938e

rsc.li/pccp

\title{
Controlling amphipathic peptide adsorption by smart switchable germanium interfaces $\dagger$
}

\author{
Laura-Marleen Baumgartner, ${ }^{a}$ Andreas Erbe, ${ }^{\text {ab }}$ Aimee L. Boyle (iD ${ }^{c}$ and \\ Martin Rabe (D)*a
}

\begin{abstract}
The in situ control of reversible protein adsorption to a surface is a critical step towards biofouling prevention and finds utilisation in bioanalytical applications. In this work, adsorption of peptides is controlled by employing the electrode potential induced, reversible change of germanium (100) surface termination between a hydrophobic, hydrogen terminated and a hydrophilic, hydroxyl terminated surface. This simple but effective 'smart' interface is used to direct adsorption of two peptides models, representing the naturally highly abundant structural motifs of amphipathic helices and coiled-coils. Their structural similarity coincides with their opposite overall charge and hence allows the examination of the influence of charge and hydrophobicity on adsorption. Polarized attenuated total reflection infrared (ATR-IR) spectroscopy at controlled electrode potential has been used to follow the adsorption process at physiological $\mathrm{pH}$ in deuterated buffer. The delicate balance of hydrophobic and electrostatic peptide/surface interactions leads to two different processes upon switching that are both observed in situ: reversible adsorption and reversible reorientation. Negatively charged peptide adsorption can be fully controlled by switching to the hydrophobic interface, while the same switch causes the positively charged, helical peptide to tilt down. This principle can be used for 'smart' adsorption control of a wider variety of proteins and peptides and hence find application, as e.g. a bioanalytical tool or functional biosensor.
\end{abstract}

\section{Introduction}

Electro-responsive interfaces alter their properties in response to an electric potential trigger. Such interfaces are an effective approach to reversibly control protein and peptide adsorption on interfaces. ${ }^{1-8}$ Hence, such 'smart' interfaces offer exciting possibilities for applications in, for instance, microfluidics, separation systems, biosensors and analytics. ${ }^{9-14}$ An especially interesting application is the employment of such hydrophobic/hydrophilic switchable interfaces to trigger and study protein adsorption, which can lead to important new insights. ${ }^{7,8}$ However, little is known about the protein dynamics that govern the adsorption and desorption upon the sudden switch of the surface properties.

\footnotetext{
${ }^{a}$ Department of Interface Chemistry and Surface Engineering, Max-Planck-Institut für Eisenforschung GmbH, Max-Planck-Str. 1, 40237 Düsseldorf, Germany. E-mail:m.rabe@mpie.de

${ }^{b}$ Department of Materials Science and Engineering, NTNU, Norwegian University of Science and Technology, 7491 Trondheim, Norway

${ }^{c}$ Leiden Institute of Chemistry, Leiden University, Einsteinweg 55, 2333 CC Leiden, The Netherlands

$\dagger$ Electronic supplementary information (ESI) available: Supporting spectroscopic data and analysis; parameters for quantitative spectra analysis. See DOI: 10.1039/ d1cp03938e
}

A promising approach for the control of protein adsorption is to exploit the nature of the sorbent surface, in particular the charge and the hydrophobicity, as these parameters have a major influence on the number, conformation and orientation of adsorbed proteins. ${ }^{15,16}$ Accordingly, on a hydrophobic/hydrophilic switchable interface in specific cases structural changes of adsorbed proteins or even reversible adsorption is to be expected. Such interfaces can be prepared for instance from self-assembled monolayers (SAMs) of long chain alkanethiols with charged terminal groups ${ }^{1,2,17}$ or brushed polymers. ${ }^{5}$ For instance a theoretical study of lysozyme interactions with a hydrophobic/hydrophilic switchable SAM revealed the influence of the surface hydrophilicity on the affinity and the surface orientation of the protein. ${ }^{7}$ In recent experimental work a hydrophobic/hydrophilic switchable interface has been used to study reversible amyloid- $\beta$ adsorption and aggregation. ${ }^{8}$ Nevertheless, experimental studies that reveal in situ structural details or the dynamics of adsorbed proteins reacting to switchable interfaces are lacking. This may be in part owing to the chemical complexity of most of these interfaces, which complicates the interpretation of data from powerful in situ structural analysis methods.

To overcome these issues, the (100) surface of germanium is used here, because of its ability to reversibly switch between 


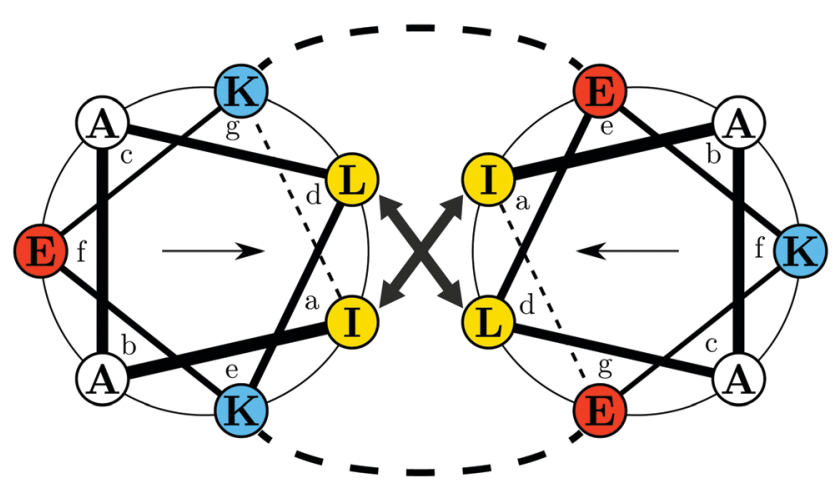

Fig. 1 Helical wheel projection of the EK coiled coil complex showing a single heptad for $\mathbf{K}$ (left): IAALKEK and $\mathbf{E}$ (right): IAALEKE. Colors indicate polarity of the amino acids at $\mathrm{pH} 7.4$ : yellow - hydrophobic; blue positively charged; red - negatively charged. Arrows inside the respective wheel projection indicate directions of the hydrophobic moments. The projection was generated with DrawCoil 1.0. ${ }^{38}$

hydroxylated and hydrogenated surface termination dependent on the applied electrode potential. ${ }^{18-23}$ It was observed that the hydrogenated or H-terminated interface is very hydrophobic. ${ }^{19}$ The change in hydrophobicity occurring with the termination switch manifests itself in the displacement of interfacial water, ${ }^{24}$ and is effective over a wide $\mathrm{pH}$ range. ${ }^{25}$ Here, the hydrophobicity change was exploited as a 'smart' interface to reversibly control changes in the adsorption behaviour of peptides at physiological $\mathrm{pH}$. The other advantage of germanium is that because of its IR-transparency, it can be used as the internal reflection element in ATR-IR spectroscopy. This method allows in situ quantification, structural analysis and orientational analysis of adsorbates from polarised spectra. ${ }^{26-28}$ Furthermore, its applicability in fibre coupled probes, flow cells, micro- or nanochannel cells makes it interesting for smart sensor applications. ${ }^{29-31}$

To demonstrate the potential of the approach for protein adsorption studies, two complementary peptides, termed $\mathbf{E}$ and $\mathbf{K}$ (Fig. 1), from the naturally abundant and versatile class of coiled-coils are used as models. ${ }^{32}$ The choice is based on the specific versatility of the EK pair. Next to their complex formation abilities they also form monomeric amphipathic $\alpha$-helices upon adsorption to hydrophobic/hydrophilic interfaces. ${ }^{33-35}$ In this state, $\mathbf{K}$ can be understood as an amphipathic class A helix which is a common lipid membrane interacting motif. ${ }^{36,37}$ Furthermore, the two peptides' primary structures are very similar. In the heptad repeating units $\mathbf{E}$ and $\mathbf{K}$ differ only in distribution and number of lysine and glutamic acid residues in the $e, f$ and $g$. positions. This difference results in an opposite overall charge, at neutral $\mathrm{pH}$. In the $\alpha$-helical conformation the charges are inversely distributed on the hydrophilic faces of the $\mathbf{E}$ and $\mathbf{K}$ helices (cf. Fig. 1). Thus, this pair allows for a study of the effects of adsorbate charge on the interfacial interactions of otherwise very similar primary and secondary structures. This is important because on an electrically triggered switching surface the charge changes, which may complicate the differentiation between effects caused by charge or by hydrophobicity.
First, the termination change of the Ge(100) surface under physiological $\mathrm{pH}$ in deuterated buffer as a function of electrode potential is studied. Next, the potential dependent adsorption of $\mathbf{E}, \mathbf{K}$, and the coiled-coil EK is studied by in situ ATR-IR spectroscopy. From these experiments surface concentration and order parameters are derived. Their analysis is then used to establish a structural model of the potential-dependent rearrangements in the adsorbed peptide layers.

\section{Results and discussion}

\subsection{Switching the germanium surface termination between -OD and -D}

First, the electrochemical behavior of the germanium interface in the peptide free buffer was examined. Deuterated phosphate buffer (d-PB) at physiological pD 7.4 was used, to enable monitoring of the amide I' bands by means of IR spectroscopy at a later stage. The cyclic voltammogram (CV) shows rising anodic current at $c a$. $-0.3 \mathrm{~V}$ assigned to oxide formation. ${ }^{20-22}$ Cathodic peaks have been assigned to the change in the surface termination from a hydrophilic $\mathrm{OH}(\mathrm{OD})$ to a hydrophobic $\mathrm{H}(\mathrm{D})$ termination. ${ }^{18-20,22}$ Here an increased cathodic current plateau was observed at $c a$. $-1.0 \mathrm{~V}$ along with minor shoulders around $-0.75 \mathrm{~V}$ and $-0.55 \mathrm{~V}$ (Fig. 2). In a $\mathrm{pH}$ dependent study in $\mathrm{ClO}_{4}{ }^{-}$ electrolyte, ${ }^{25}$ the reductive surface transformation was reported at $-0.7 \mathrm{~V}$ for $\mathrm{pH} 8$, which is in range of the cathodic currents observed here. Here, the respective transformation should mainly be the deuterated equivalent, according to:

$$
\mathrm{Ge}-\mathrm{OD}+2 \mathrm{D}^{+}+2 \mathrm{e}^{-} \rightleftharpoons \mathrm{Ge}-\mathrm{D}+\mathrm{D}_{2} \mathrm{O}
$$

The equivalence of the surface termination change in $\mathrm{H}_{2} \mathrm{O}$ and $\mathrm{D}_{2} \mathrm{O}$ is confirmed by in situ ATR-IR spectroscopy with potential step experiments. Because of the isotope effect the position of Ge-D bands is expected to be shifted approximately by a factor of $\frac{1}{\sqrt{2}}$ with respect to the Ge-H bands. Accordingly, the spectral region around $2000 \mathrm{~cm}^{-1}$, where the $\mathrm{Ge}-\mathrm{H}_{1}$ and the Ge- $\mathrm{H}_{2}$ stretching modes are observed, ${ }^{20-23,25,39}$ ( $c f$. overview of the reference values in ESI, $\dagger$ Table S1) is found free of features (cf. ESI, $\dagger$ Fig. S1). However, a broad peak in the range $1350 \mathrm{~cm}^{-1}$ to $1550 \mathrm{~cm}^{-1}$ grows at decreasing potentials steps, i.e. during the negative scan, starting below $-0.59 \mathrm{~V}$. The peak at the lowest potential $-1.19 \mathrm{~V}$ is depicted in Fig. $2 \mathrm{~b}$. In the subsequent positive scan, the peak decreases showing the reversibility of the termination change, whereas at the final potential of $-0.19 \mathrm{~V}$ some absorbance remains ( $c f$. ESI, $\dagger$ Fig. S1). The potential dependent absorbance in this region was analysed further by a singular value decomposition-based matrix least square (SVD-MLS) algorithm ${ }^{40,41}$ (details are discussed in ESI, $\dagger$ Section S1.1). The analysis reveals that two independent spectral components grow or shrink independently from each other with the potential variation (Fig. $2 \mathrm{~b}$ ). The component centered around $1430 \mathrm{~cm}^{-1}$ to $1450 \mathrm{~cm}^{-1}$ is assigned to the HOD bending vibration $\delta_{\mathrm{HOD}}{ }^{42}$ It arises from an increasing $\mathrm{H}_{2} \mathrm{O}$ 

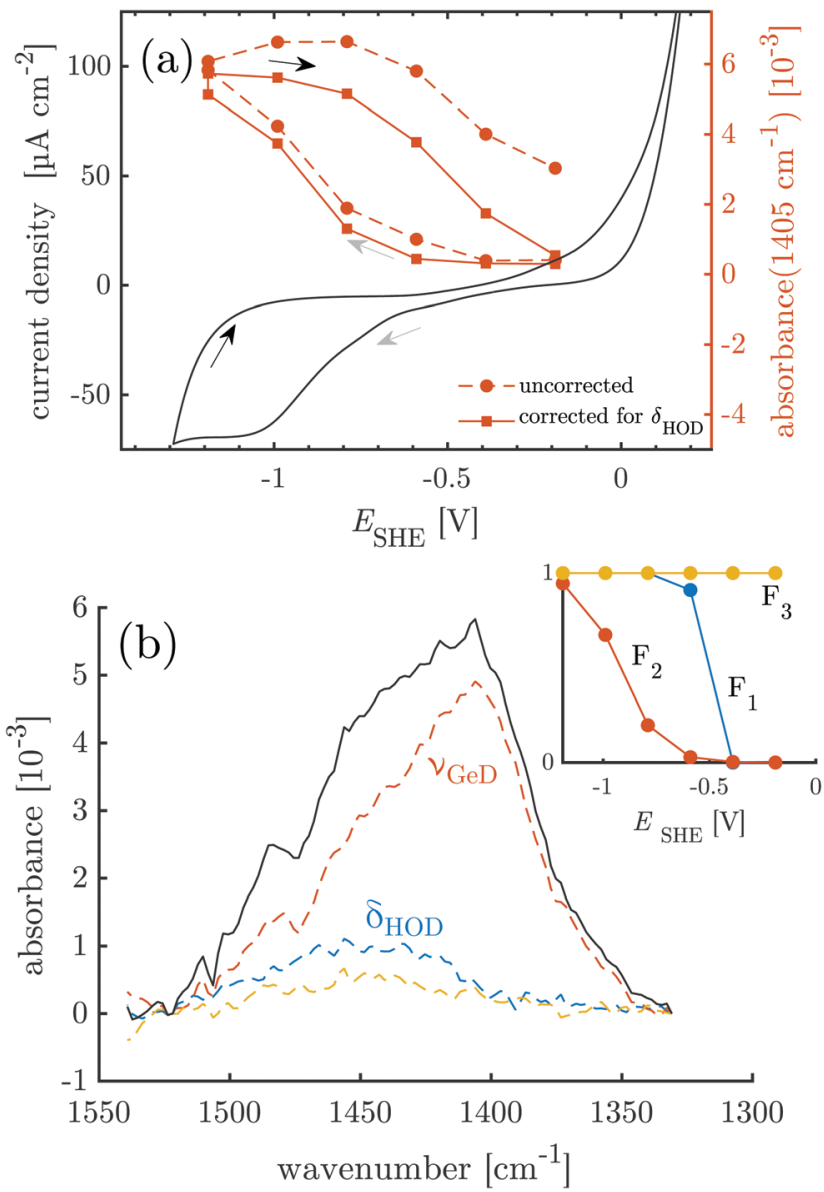

Fig. 2 Overlay of CV data and results of potential dependent ATR-IR spectroscopy. (a) Intensity of the Ge- $\mathrm{D}_{1}$ stretching mode band (uncorrected and corrected for contribution of HOD bending mode) and the cyclic voltammogram measured at $10 \mathrm{mV} \mathrm{s}^{-1}$. (b) Absorbance in $\mathrm{p}$-polarisation in the Ge-D stretching mode region $\left(1330 \mathrm{~cm}^{-1}\right.$ to $\left.1550 \mathrm{~cm}^{-1}\right)$ at $-1.19 \mathrm{~V}$. Colored dashed lines and inset are the results of the SVD-MLS analysis described in detail in ESI, $\uparrow$ Section S1.1. The resulting independent spectral components of the negative scan are labeled with their assignments. The inset shows their associated dependencies on the decreasing electrode potential.

content. The second component with a peak at $1395 \mathrm{~cm}^{-1}$ to $1418 \mathrm{~cm}^{-1}$ is a combined Ge-D stretching vibration band $\nu_{\mathrm{GeD}}$ and contains contributions from $\mathrm{GeD}_{1}$ and $\mathrm{GeD}_{2}$ groups.

The $\left(\delta_{\mathrm{HOD}}\right)$ component increases over the course of the full experiment independent of the potential scan direction (see ESI, $\dagger$ Fig. S2-S5), showing that trace $\mathrm{H}_{2} \mathrm{O}$ is constantly introduced into the experimental setup from an external source. The ATR-IR spectra were corrected for the disturbing influence of HOD by recombining the components found by SVD-MLS and omitting the $\delta_{\mathrm{HOD}}$ components (ESI, $\dagger$ Fig. S6). As discussed in detail in ESI, $\uparrow$ Section S1.1, the main peak of $\nu_{\mathrm{GeD}}$ at $1405 \mathrm{~cm}^{-1}$ is assigned to the $\mathrm{GeD}_{1}$ stretching vibration, while the high frequency shoulder visible in Fig. $2 \mathrm{~b}$ is assigned to $\mathrm{GeD}_{2}$. So far surface GeD bands have been reported in UHV experiments only. ${ }^{43,44}$ Here, the ATR-IR spectra confirm the expectation that the termination change of the Ge-interface happens analogously in $\mathrm{H}_{2} \mathrm{O}$ and $\mathrm{D}_{2} \mathrm{O}$ buffers at physiological $\mathrm{pH}$, which is
Table 1 Characteristic values for peptide surface concentration $\Gamma_{\text {pep }}$, order parameter $S_{\theta}$ and average angle $\theta$ under specific germanium surface conditions

\begin{tabular}{|c|c|c|c|c|}
\hline & $\begin{array}{l}\text { Surface state scan } \\
\text { direction } E_{\mathrm{SHE}}[\mathrm{V}]\end{array}$ & $\begin{array}{c}\text { Hydrophilic } \\
\downarrow \\
-0.59\end{array}$ & $\begin{array}{c}\text { Hydrophobic } \\
\uparrow \\
-1.19\end{array}$ & $\begin{array}{c}\text { Hydrophilic } \\
\uparrow \\
-0.19\end{array}$ \\
\hline \multirow{4}{*}{$\mathbf{K}$} & $\Gamma_{\text {pep }}\left[\mathrm{pmol} \mathrm{\textrm {cm } ^ { - 2 } ]}\right.$ & $31.0 \pm 4.5$ & $46.8 \pm 5.8$ & $66.7 \pm 8.5$ \\
\hline & $S_{\theta}$ & $0.34 \pm 0.13$ & $-0.21 \pm 0.09$ & $0.07 \pm 0.10$ \\
\hline & $\theta\left[^{\circ}\right]$ & $41.7 \pm 5.0$ & $63.9 \pm 4.6$ & $52.0 \pm 3.8$ \\
\hline & $\Gamma_{\text {pep }}\left[\mathrm{pmol} \mathrm{\textrm {cm } ^ { - 2 } ]}\right.$ & $0.0 \pm 0.7$ & $30.0 \pm 3.7$ & $5.1 \pm 1.6$ \\
\hline \multirow[t]{2}{*}{$\mathbf{E}$} & $\begin{array}{l}S_{\theta} \\
\theta\left[{ }^{\circ}\right]\end{array}$ & $-^{a}$ & $\begin{aligned}-0.36 & \pm 0.11 \\
72.2 & \pm 7.3\end{aligned}$ & $-^{a}$ \\
\hline & $\Gamma_{\text {pep }}\left[\mathrm{pmol} \mathrm{cm}^{-2}\right]$ & $28.8 \pm 3.7$ & $45.4 \pm 5.7$ & $18.8 \pm 2.8$ \\
\hline EK & $\begin{array}{l}S_{\theta} \\
\theta\left[{ }^{\circ}\right]\end{array}$ & $\begin{aligned}-0.13 & \pm 0.09 \\
60.2 & \pm 4.0\end{aligned}$ & $\begin{aligned}-0.26 & \pm 0.10 \\
66.4 & \pm 5.1\end{aligned}$ & $\begin{aligned}-0.06 & \pm 0.09 \\
57.3 & \pm 3.8\end{aligned}$ \\
\hline
\end{tabular}

${ }^{a}$ Value not reported because of zero or low absorbance.

a prerequisite for the IR spectroscopic study of proteins with this switchable system.

The potential dependent, corrected absorbance in p-polarisation at $1405 \mathrm{~cm}^{-1} A_{1405}$ indicates the degree of D-termination of the surface (Fig. 2a) and confirms the assignment of the reductive currents in the $\mathrm{CV}$ to the $\mathrm{Ge}-\mathrm{OD} \rightleftharpoons \mathrm{Ge}-\mathrm{D}$ termination change. Furthermore, it shows that within the chosen potential region the termination change is reversible with a hysteresis, in accordance with similar observations for the Ge-H termination..$^{20,23}$ In the following, the potential dependent termination change of the germanium interface is exploited as a switching process between a hydrophobic and a hydrophilic interface. In this regard the hysteresis of $A_{1405}$ means that during the negative scan the surface remains hydrophilic down to $-0.59 \mathrm{~V}$. However, during the positive scan a similar GeOD coverage and hence a comparable hydrophilicity is not reached below $-0.19 \mathrm{~V}$ (see also Table 1 ).

\subsection{Peptide adsorption can be controlled by switching the germanium surface termination}

Next, the hydrophobic/hydrophilic switching germanium interface was used to study peptide adsorption. Potential dependent ATR-IR spectra of $\mathbf{E}, \mathbf{K}$ solutions and their mixture, i.e. the coiled-coil complex EK show additional bands originating from adsorbed peptides. These are the amide $\mathrm{I}^{\prime}$ bands with maxima in the range $1635 \mathrm{~cm}^{-1}$ to $1645 \mathrm{~cm}^{-1}$, the antisymmetric $\mathrm{COO}^{-}$ stretch of the deprotonated glutamic acid side chains at ca. $1560 \mathrm{~cm}^{-1}$, and the broad amide $\mathrm{II}^{\prime}$ band centered between $1440 \mathrm{~cm}^{-1}$ to $1460 \mathrm{~cm}^{-1}$ (Fig. 3, 4 and 5). ${ }^{45}$ These spectra are well in accordance with transmission spectra of $\mathbf{E}$ and $\mathbf{K}$ peptides. $^{34}$ Additionally, at the low frequency flank of the amide $\mathrm{II}^{\prime}$, the GeD band appeared with the maximum at ca. $1405 \mathrm{~cm}^{-1}$, which as discussed in Section 2.1 is assigned to the surface termination change. In general, the peptide modes increase in absorbance with decreasing potential. The peptide concentration in the experiment was set low enough to detect adsorbed peptide only, i.e., without contributions from bulk peptide. This was confirmed by the absence of amide $\mathrm{I}^{\prime}$ bands after injection of non-adsorbing $\mathbf{E}$. In contrast $\mathbf{K}$ and EK, at the same concentrations, already started to adsorb at open 

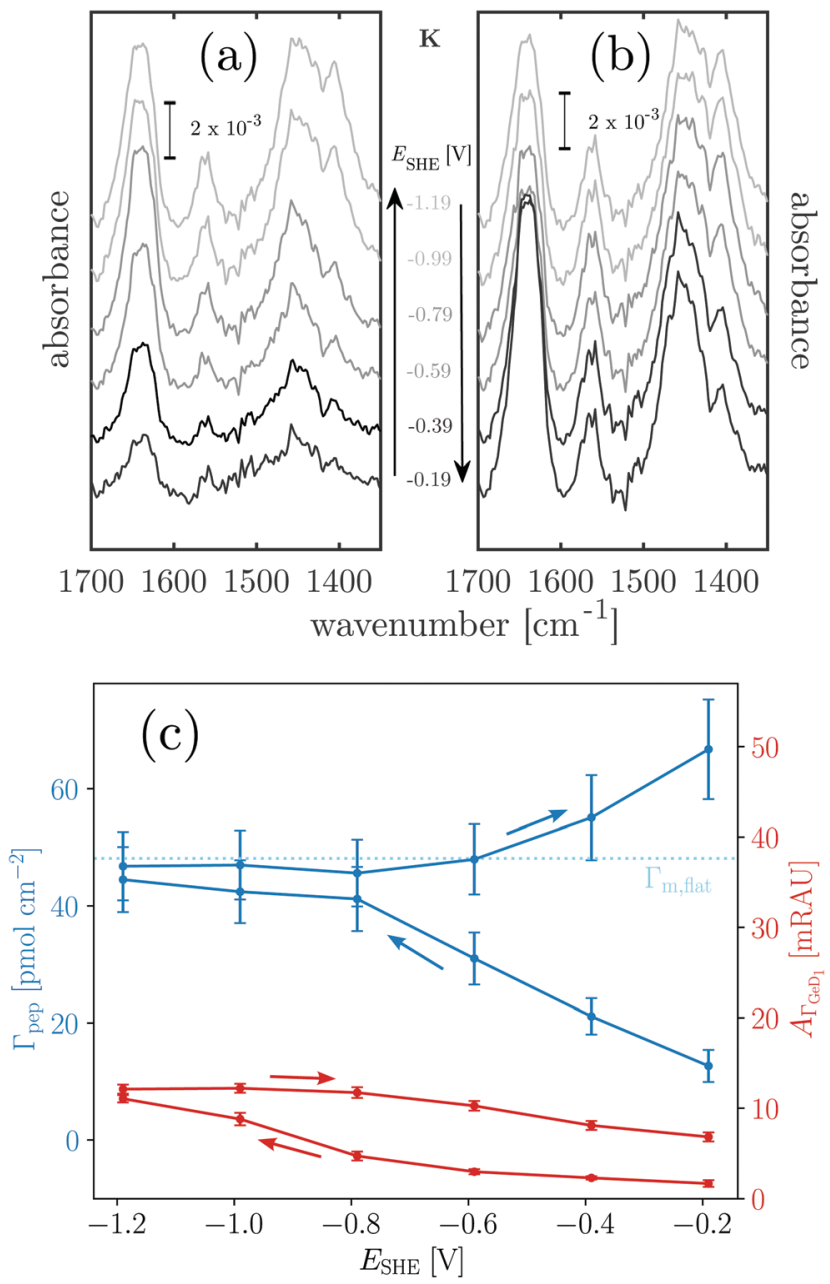

Fig. 3 Potential dependent ATR-IR spectroscopy of $\mathbf{K}$. Spectra in $\mathrm{p}$-polarisation in the amide range during (a) negative scan and (b) positive scan. (c) Potential dependency of peptide surface concentration $\left(\Gamma_{\text {pep }}\right)$ and surface concentration proportional absorbance of the $\mathrm{GeD}_{1}$ stretching vibration $\left(A_{\Gamma_{\mathrm{GeD}_{1}}}\right)$. Arrows indicate the scan directions. $[\mathrm{K}]=3.6 \mu \mathrm{M}$, in $\mathrm{d}-\mathrm{PB}, \mathrm{pD}=7.4$.

circuit after injection. The amide $\mathrm{I}^{\prime}$ bands show a clear asymmetry originating from more than a single underlying component as discussed in Section 2.3.

The peptide surface concentration $\Gamma_{\text {pep }}$ was determined from the integral of the amide $\mathrm{I}^{\prime}$ band and an integrated absorption coefficient from former measurements (eqn (6)). ${ }^{34}$ To examine the correlation between peptide adsorption and surface hydrophobicity, the surface concentration proportional absorbance $A_{\Gamma_{\mathrm{GeD}_{1}}}$ of the $\nu_{\mathrm{GeD} 1}$ at $1405 \mathrm{~cm}^{-1}$ was also calculated from the polarised spectra (eqn (3)). Note that, this time the deconvolution of the spectra in the region around $1450 \mathrm{~cm}^{-1}$ was not feasible due to the additional contributions from the amide $\mathrm{II}^{\prime}$ band in the range between $1460 \mathrm{~cm}^{-1}$ to $1490 \mathrm{~cm}^{-1}{ }^{45}$ For all experiments with peptides present, the $A_{\Gamma_{\mathrm{GeD}_{1}}}$ hysteresis indicates that the termination change from GeOD to GeD and hence the switching between hydrophilic and hydrophobic interface is unaltered compared to the peptide free experiment (Fig. 3b-5b).
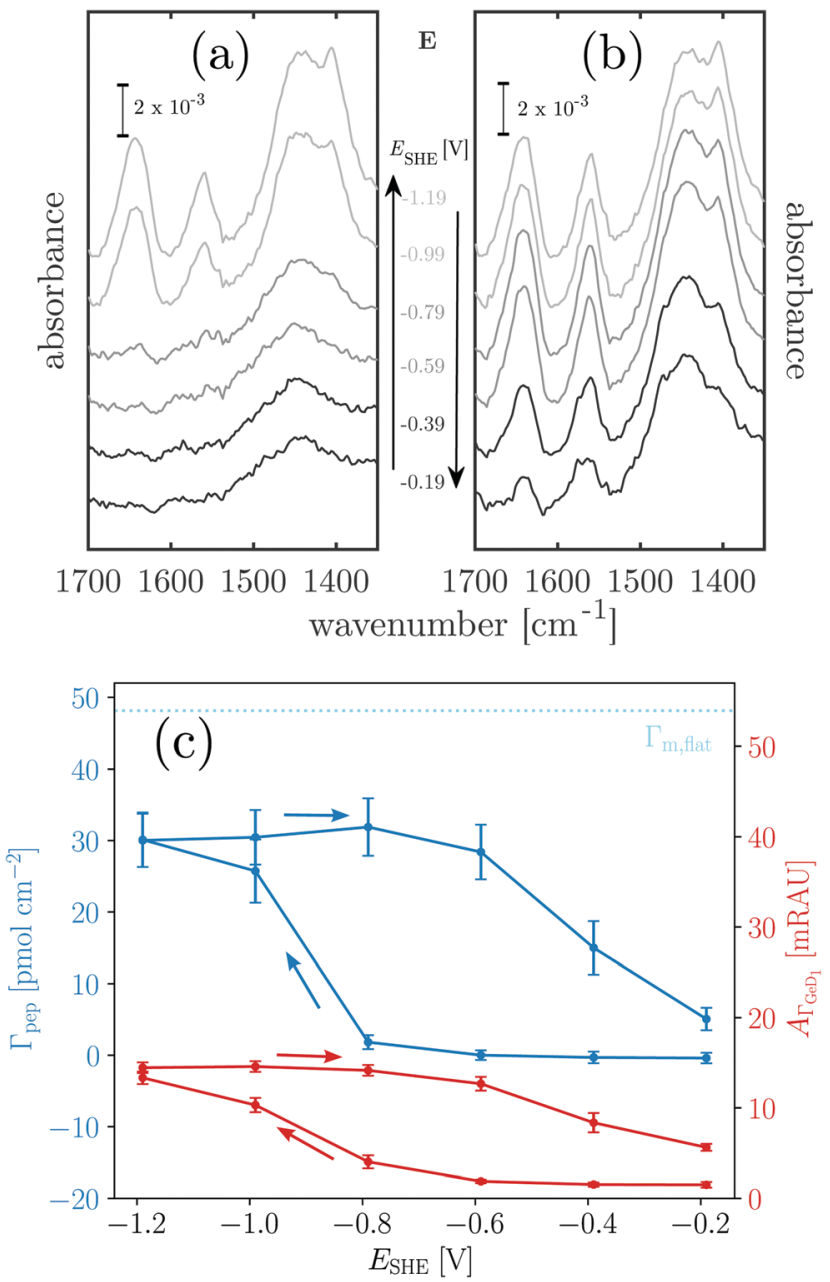

Fig. 4 Potential dependent ATR-IR spectroscopy of E. Spectra in ppolarisation in the amide range during (a) negative scan and (b) positive scan. (c) Potential dependency of peptide surface concentration $\left(\Gamma_{\text {pep }}\right)$ and surface concentration proportional absorbance of the $\mathrm{GeD}_{1}$ stretching vibration $\left(A_{\Gamma_{\mathrm{GeD}_{1}}}\right)$. Arrows indicate the scan directions. [E] $3.6 \mu \mathrm{M}$, in d-PB, $\mathrm{pD}=7.4$.

Positively charged $\mathbf{K}$ readily adsorbs at the hydrophilic negatively charged interface during decreasing and during increasing potentials above $-0.79 \mathrm{~V}$, as indicated by the potential dependency of $\Gamma_{\text {pep }}$ (Fig. 3). However, at the hydrophobic interface the adsorption of $\mathbf{K}$ is stopped as $\Gamma_{\text {pep }}$ stagnates from $-0.79 \mathrm{~V}$ to $-1.19 \mathrm{~V}$. Electrostatic interactions favor the adsorption at the hydrophilic germanium, while these interactions are counteracted at the hydrophobic interfaces, possible reasons for this are discussed in Section 2.4. The potential dependent plot shows the time averaged peptide concentrations from several spectra measured per potential step. The time dependency of $\Gamma_{\text {pep }}$ was also investigated (ESI, $\dagger$ Fig. S7) and reveals that $\mathbf{K}$ adsorbs at a constant rate, without a sign of saturation within the scope of this experiment.

To estimate the surface coverage of peptide monolayers, two special case models of perfectly ordered monolayers of hard cylinders (diameter: $1 \mathrm{~nm}$, length: $23 \times 0.15 \mathrm{~nm}$ ) are considered. These models yield maximum monolayer surface concentrations 


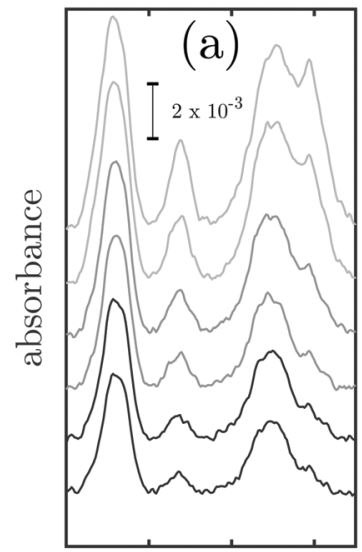

1700160015001400

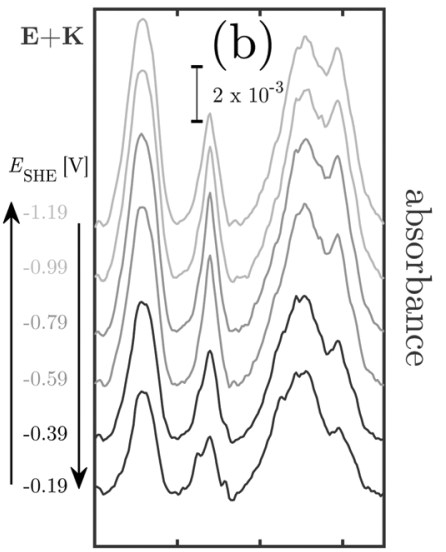

1700160015001400 wavenumber $\left[\mathrm{cm}^{-1}\right]$

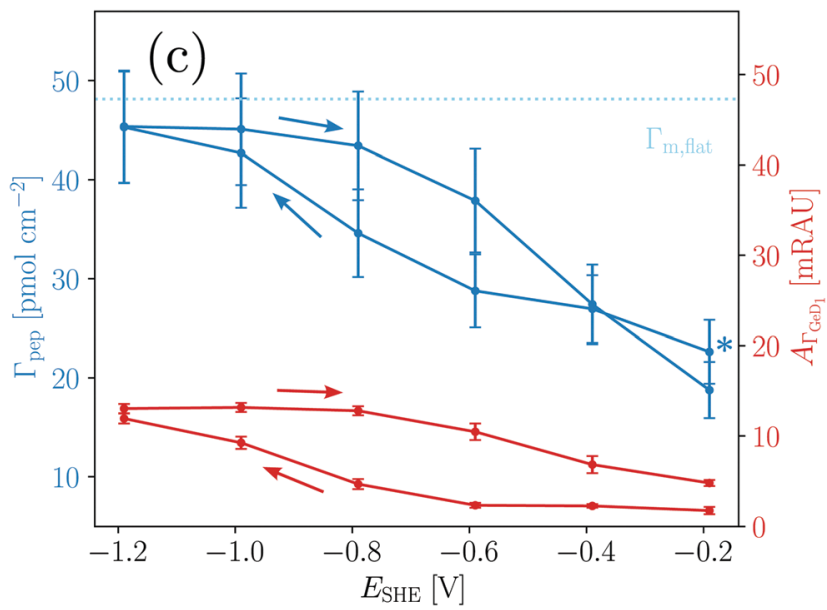

Fig. 5 Potential dependent ATR-IR spectroscopy of EK. Spectra in p-polarisation in the amide range during (a) negative scan and (b) positive scan. (c) Potential dependency of peptide surface concentration $\left(\Gamma_{\text {pep }}\right)$ and surface concentration proportional absorbance of the $\mathrm{GeD}_{1}$ stretching vibration $\left(A_{\Gamma_{\mathrm{GeD}_{1}}}\right)$. The asterisk indicates the initial datapoint during upscan. Arrows indicate the scan directions. [EK] $3.6 \mu \mathrm{M}$, in d-PB, $\mathrm{pD}=7.4$

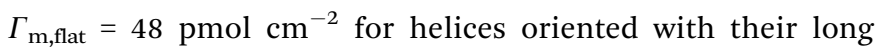
(molecular) axis parallel to the interface and $\Gamma_{\mathrm{m} \text {,up }}=$

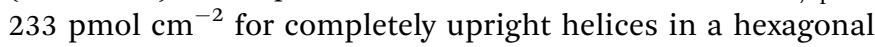
packing. However, these values must be understood as absolute maximum limiting surface concentrations. Surface irregularities, side chain contributions, helix irregularities and inter-chain interactions are not considered and perfect, gap filling order is assumed. The surface concentration measured for $\mathbf{K}$ on the hydrophobic surface is ca. $47 \mathrm{pmol} \mathrm{cm}^{-2}$ (Table 1). Thus, the interface is already densely covered with peptide when the termination change occurs, which could explain why no significant further hydrophobic adsorption is observed. The recommencing, electrostatically driven adsorption during the increasing potential steps leads to an increase in surface concentrations significantly above $\Gamma_{\mathrm{m} \text {,flat }}$, but still below $\Gamma_{\text {m,up }}(c f$. Table 1 and Fig. 3 ). Thus, either multilayer formation or a densification by changing to more upright oriented helices occurs. The latter will be elucidated by an analysis of the peptides' order parameters and its secondary structure in Sections 2.3 and 2.4.

Negatively charged $\mathbf{E}$ shows a completely different behaviour than K. The hydrophilic interface remains free of $\mathbf{E}$ during the negative scan, but adsorption is instantaneously triggered by the increase of hydrophobicity occurring at $-0.79 \mathrm{~V}$ (Fig. 4c). Comparison with $\Gamma_{\mathrm{m} \text {,flat }}$ indicates that a peptide monolayer is formed, at the hydrophobic interface where the surface concen-

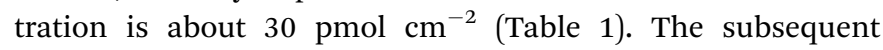
potential increase that causes increasing hydrophilicity triggers the desorption of E. Remarkably, the hysteresis of the $\mathbf{E}$ surface concentration largely follows the hysteresis of the Ge-D surface concentration (Fig. 4b), proving their direct correlation. On the hydrophilic OD terminated interface $\mathbf{E}$ adsorption is prohibited by repulsive electrostatic interactions. These repulsive interactions must be exceeded by the hydrophobic attractive forces on the hydrophobic Ge-D terminated interface to initiate adsorption. When switched back to the hydrophilic interface at $-0.19 \mathrm{~V} \mathbf{E}$ is almost completely removed from the interface (Table 1). Hence, the ad-/desorption of $\mathbf{E}$ is triggered in a highly reversible manner by switching the hydrophobicity of the germanium interface.

When mixed in equimolar proportions the peptides form the overall neutrally-charged coiled-coil complex EK. On the hydrophilic Ge-OD interface the complex shows biphasic adsorption during the negative scan (see also ESI, $\dagger$ Fig. S7) and partial desorption during the positive scan (Fig. 5c). The desorption is incomplete and peptide remains at the interface at a submonolayer coverage (Table 1). The adsorption during the negative potential steps is biphasic, which is more obvious from the time dependent plot (ESI, $\uparrow$ Fig. S7). At the hydrophobic Ge-D interface formed below $-0.79 \mathrm{~V}$ the peptide surface concentration is almost stable with values very close

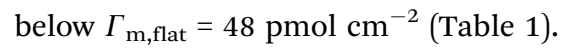

From the results with EK it cannot be determined unequivocally which of the peptides present in the equilibrium state adsorbs. Based on the folding constant of EK at $25{ }^{\circ} \mathrm{C}, K_{\mathrm{F} 25}=$ $1.77 \times 10^{7} \mathrm{l} \mathrm{mol}^{-1},{ }^{46}$ approximately $88 \%$ of the peptide in solution is folded in the coiled-coil complex, while the rest is in a monomeric state. It is conceivable that EK adsorbs to the hydrophilic, charged Ge-OD terminated interface orienting itself to maximize attractive electrostatic forces. On the other hand $\mathbf{E}$ and $\mathbf{K}$ may adsorb as monomers in similar fashions as they do when present alone in solution. The significant desorption during the positive scan, following the hysteresis of $A_{\Gamma_{\mathrm{GeD}_{1}}}$ is comparable to the behaviour of $\mathbf{E}$ alone and hence indicates that among other processes hydrophobically attached, monomeric, helical $\mathbf{E}$ desorbs.

\subsection{Amide $I^{\prime}$ band shape analysis confirms helicity of adsorbed $\mathbf{E}$ and $\mathbf{K}$}

The amide $\mathrm{I}^{\prime}$ bands of all peptides show specific shapes that indicate several underlying peaks with different peak frequencies. Furthermore, the band shapes vary with potential and light 
polarisation. Consequently, the transition dipole moments $M$ of the different underlying amide $I^{\prime}$ vibrations differ in their orientation with respect to the interface. For a detailed analysis, an automated fitting routine was established, in which sums of three Gaussians were employed, resulting in a proper description of the band shapes (ESI, $\dagger$ Fig. S8-S11). The bands are dominated by two main peaks at $1631 \mathrm{~cm}^{-1}$ to $1634 \mathrm{~cm}^{-1}$ and $1649 \mathrm{~cm}^{-1}$, termed $\nu_{\mathrm{a}}$ and $\nu_{\mathrm{b}}$ respectively, in the following. The variations of the intensities of these two main components with respect to each other mainly cause the potential and polarisation dependent shape variations of the amide $\mathrm{I}^{\prime}$. A third, small component centered at $1668 \mathrm{~cm}^{-1}$ was necessary to adequately describe the band shapes at the high frequency flank. This minor high frequency component here is assigned to C-terminal primary amide $\mathrm{CONH}_{2},{ }^{47}$ which is generally observed between $1670 \mathrm{~cm}^{-1}$ to $1690 \mathrm{~cm}^{-1}$ in solution. Similar band shapes and patterns were also observed for homo- and hetero coiled-coil bound $\mathbf{E}$ and $\mathbf{K}$ as well as when bound to lipid membranes as monomeric $\alpha$-helices. ${ }^{33,34}$ The similarity of the amide I' spectra of the different forms arises from the same molecular origin which is the solvation of the amides in amphipathic helices. ${ }^{48}$

Solvent inaccessible amides at hydrophobic interfaces as in the interior of the coiled-coil or buried in the hydrophobic part of a lipid monolayer show relatively sharp amide bands centered around $1650 \mathrm{~cm}^{-1}\left(\nu_{\mathrm{b}} ; \mathrm{b}\right.$ as in buried). This band is also commonly assigned to buried $\alpha$-helical segments in proteins. ${ }^{49}$ However, solvent accessible amides as they appear in a large fraction on the outside of the coiled-coil or the solvent accessible face of adsorbed peptide, show broader, significantly red shifted bands around $1630 \mathrm{~cm}^{-1}\left(\nu_{\mathrm{a}}\right.$; a as in accessible) owing to additional hydrogen bonding between water and the amide carbonyl. ${ }^{48,50,51}$ In the studied system a disordered monomeric peptide would be expected to yield a broad contribution centered around $1645 \mathrm{~cm}^{-1} \cdot{ }^{34,49}$ An unordered component might also contribute to the spectra measured here, but cannot unequivocally be resolved by peak fitting, owing to the strong overlap with the other two major components. Nevertheless, the finding of the two major components indicates the presence of solvent inaccessible and solvent accessible helix amides and thus, of hydrophobically bound helices in the adsorbed peptide layers.

The different hydrogen bonding situation of the amides in amphipathic helices also leads to a different orientation of the carbonyl bond with the solvent accessible carbonyls having a higher angle with the helix axis. ${ }^{48}$ This higher tilt, together with the different binding situation caused by the additional hydrogen bond must result in different angles $\alpha$, between the components' $M$ and the helix' long axis. The different $\alpha$ values would explain the observed variations of the band shape with light polarisation. To test this hypothesis, the order parameters of the $M$ for the main components $\nu_{\mathrm{a}}$ and $\nu_{\mathrm{b}}$ were calculated by eqn (9) using $\alpha=0$ and their average angle to the interface normal $\theta_{\mathbf{M}}$ was determined by eqn (10) (ESI, $\dagger$ Fig. S12). The values for $\nu_{\mathrm{a}}$ and $\nu_{\mathrm{b}}$ differ in the order of $5^{\circ}$ to $15^{\circ}$ for $\mathbf{E}$ and $\mathbf{K}$, which supports our assignments of the observed amide $\mathrm{I}^{\prime}$ components. The difference in the case of EK is less pronounced which may be caused by a less uniform adsorption.

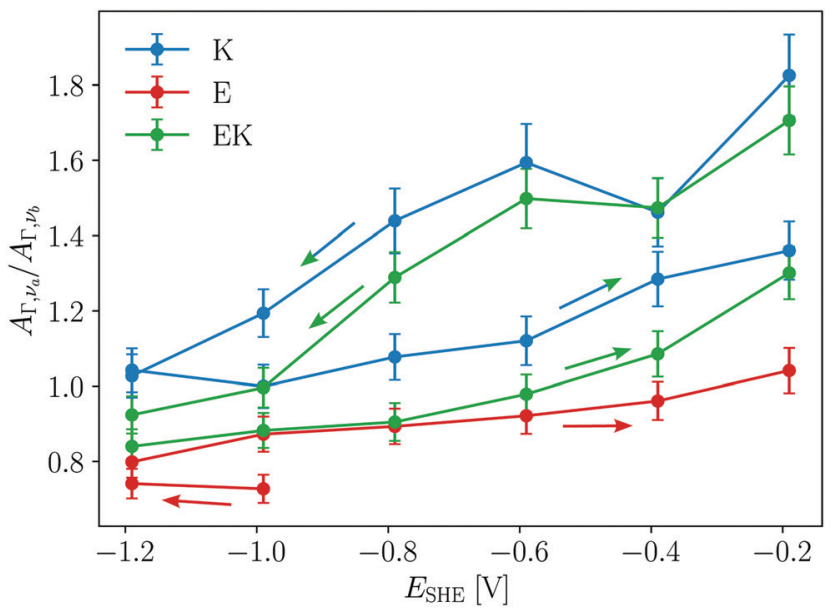

Fig. 6 Ratio of absorbance of solvent accessible $\left(A_{\Gamma, \nu}\right)$ to buried amides $\left(A_{\Gamma, \nu_{\mathrm{b}}}\right)$ versus potential. Arrows indicate the scan directions.

Hydrophobic peptide adsorption of E, K, and EK was accompanied by a reduction of the number of solvent accessible amides. This is evidenced by the potential dependent ratio of the $A_{\Gamma}$ values calculated by eqn (3) for the two main components $\left(A_{\Gamma, \nu_{\mathrm{a}}} / A_{\Gamma, \nu_{\mathrm{b}}}\right)$ in Fig. 6. For $\mathbf{K}$ and EK the decrease of $A_{\Gamma, \nu_{\mathrm{a}}} / A_{\Gamma, \nu_{\mathrm{b}}}$ during the negative scan and the increase during the positive scan clearly show that the hydrophobicity change of the interface causes a change of the surface binding of the peptides towards hydrophobic interactions. In earlier transmission IR studies for KK homo- and EK hetero coiled coils $A_{\Gamma, \nu_{\mathrm{a}}} / A_{\Gamma, \nu_{\mathrm{b}}}$ values in the range of 1.8 to 2.5 were observed and helical, monomeric $\mathbf{K}$ adsorbed with its helix axis parallel to a lipid membrane interface gave values around 1 . Hence, the relatively high $A_{\Gamma, \nu_{\mathrm{a}}} / A_{\Gamma, \nu_{\mathrm{b}}}$ values above 1.4 of $\mathbf{K}$ and EK could indicate that coiled coils are adsorbed during the initial negative scan until $-0.6 \mathrm{~V}$ (Fig. 6). At the hydrophobic $\mathrm{GeH}$ terminated interface all peptides showed values around or below 1, with $\mathbf{E}$ showing the lowest values. Hence for all peptides studied here, approximately half of the amide groups per helix are inaccessible for the solvent due to the hydrophobic binding to the interface.

For $\mathbf{E}$ the presence of hydrophobically bound $\alpha$-helices in combination with the low observed adsorption to the hydrophobic Ge-D terminated interface well below $\Gamma_{\mathrm{m} \text {,flat }}$, suggests that it adsorbs as a monomeric amphipathic helix. In contrast, $\mathbf{K}$ adsorbs to the hydrophilic Ge-OD terminated interface with higher density. This means that its hydrophobic, solvent inaccessible helix amides must be buried within the peptide, which is most straightforwardly explained by homomeric coiled-coil formation. Both $\mathbf{K}$ and $\mathbf{E}$ dimer formation of varying intensities have been already observed in solution and at interfaces ${ }^{34,46}$ and can be promoted for instance by high local concentration, $\mathrm{pH}$ or ionic strength. From the folding constant of the KK homo-coils ${ }^{46}$ it can be deduced that at the concentration used here only $\mathrm{ca} .2 \%$ to $3 \%$ of the bulk molecules are folded into homo-coils. The observed spectra of $\mathbf{K}$ and the high $A_{\Gamma, \nu \mathrm{a}} / A_{\Gamma, \nu \mathrm{b}}$ ratio during the initial negative scan indicate that in 
comparison to the bulk an increased fraction of $\mathbf{K}$ molecules adsorbs as dimeric homo coiled-coils at the hydrophilic Ge-OD terminated interface. Hence, under the assumption that we observed equilibrium structures, major contributions to the adsorption free enthalpy come from the favourable hydrophobic interactions between the peptide chains in the dimers.

\subsection{Order parameters unveil orientation variations of adsorbed $\mathbf{E}$ and $\mathrm{K}$}

For a measurement of the helix order parameter $S_{\theta}$ according to eqn (9) a value for $\alpha$ is needed. However, different values in the range $20^{\circ}$ to $40^{\circ}$ have been used for the interpretation of polarised ATR-IR of helical peptides. ${ }^{52-54}$ Here, a value of $(35 \pm 5)^{\circ}$ was employed for $\nu_{\mathrm{b}}$ because this represents the value range frequently determined in dried and thus unsolvated peptide films with an amide I peak centre around $1650 \mathrm{~cm}^{-1} \cdot{ }^{52,54}$ At the hydrophobic interface $\mathbf{E}$ yields $S_{\theta}$ values of about -0.4 (Table 1 and Fig. 7a) and is very close to the minimum possible value of perfectly flat oriented helices which would give $S_{\theta}=-0.5$. Hence, the order parameter proves that $\mathbf{E}$ adsorbs as helices, oriented close to parallel to the interface
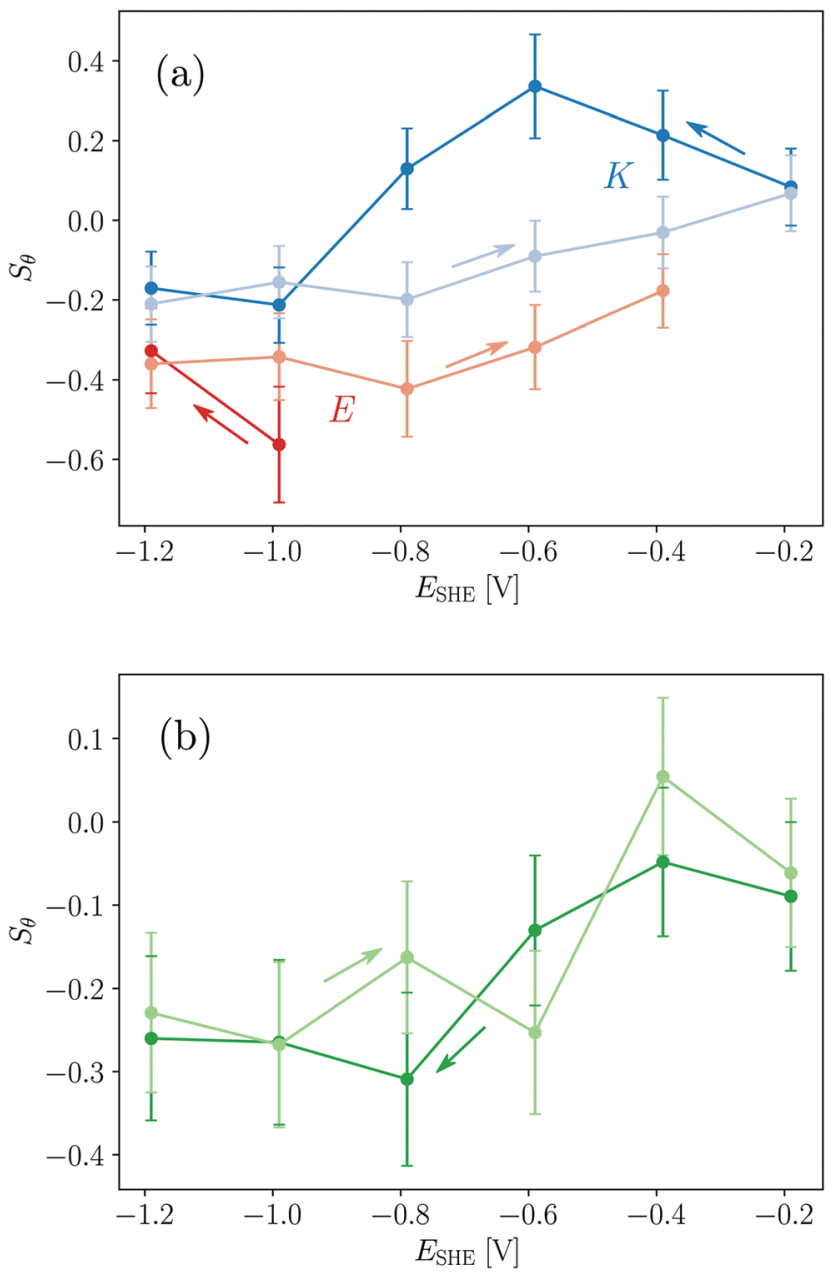

Fig. 7 Potential dependent order parameters of $\mathbf{E}, \mathbf{K}$ (a) and EK (b) determined from $\nu_{\mathrm{b}}$.
(Fig. 8). A decrease of order and a "less parallel" orientation manifest during the potential increase by $S_{\theta}$ increasing towards 0 . Thus, the increasing hydrophilicity due to increasing Ge-OD termination reduces the peptide ordering and triggers the desorption of E. In that regard the picture provided by Fig. 8 is oversimplified as it does not picture this partial loss of orientational order.

The order parameters of $\mathbf{K}$ show a more complex potential dependency and for a self-consistent model, as depicted in Fig. 8 that explains these observations, the surface concentration data for $\mathbf{K}$ must be taken into account. Starting with the negative scan, the initial increase in $S_{\theta}$ (Fig. 7a) coincides with increasing surface concentration (Fig. 3c). This indicates that peptide adsorption coincides with an increasing order in the adsorbed layer, or an upward tilt of the helical peptides. The maximum $S_{\theta}$ on the hydrophilic interface corresponds to an intermediate angle of $42^{\circ}$ (Table 1 ). The increased order and upward orientation is necessary to provide the space needed to adsorb additional molecules. The germanium termination change commencing below $-0.79 \mathrm{~V}$ stopped the adsorption ( $\Gamma$ remains constant, Fig. $3 \mathrm{~b}$ ) and caused a significant drop of the order parameter to negative values. This is interpreted as a downward tilt of a fraction of adsorbed helices to change their bound state from a homomeric KK coiled-coil to a mostly parallel oriented monomeric helix with its hydrophobic face oriented towards the germanium interface (Fig. 8). This orientation requires more surface area per molecule than the previous, on average, more upright orientation. Thus, the peptide adsorption is stopped because of the full coverage of the interface. The latter is also supported by the fact that the peptide surface concentration at the hydrophobic interface is very close to $\Gamma_{\mathrm{m} \text {,flat }}$ (Table 1$)$. When switching the interface back to hydrophilic during the upscan above $-0.79 \mathrm{~V}$, the slowly increasing order parameter is interpreted as an upward tilt of the helices (Fig. 7a). The upward tilt indicates that the loss of hydrophobic interface binding is compensated by forming again more upright KK coiled-coils. The newly available space at the interface caused by the ongoing upward tilt, causes the resurgence of the adsorption, as indicated by the increase in $\Gamma_{\text {pep }}$ (Fig. 3b). Alternatively, order parameters close to 0 could be interpreted as highly unstructured peptides or more isotropically distributed helices. However, this is not considered likely here, because the former contradicts the observation of the amide I' band's two-component shape which indicates the

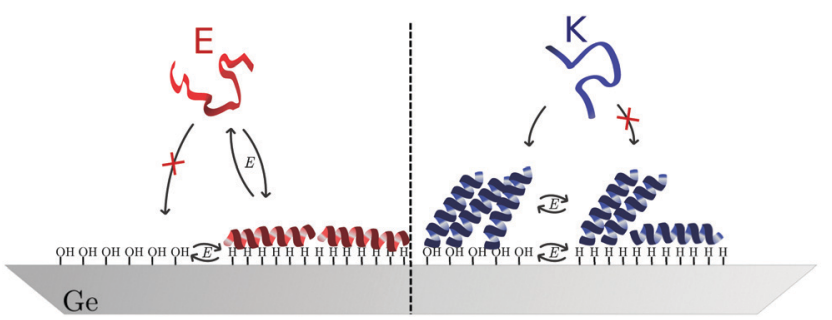

Fig. 8 Model for the potential dependent adsorption of $\mathbf{E}$ and $\mathbf{K}$ at hydrophobic/hydrophilic switchable germanium (100) interface. 
occurrence of $\alpha$-helical structures. Isotropically distributed helices on the other hand appear not very likely due to the observed increase in $\Gamma_{\text {pep }}$ which clearly requires increasing order and improved helix packing.

It is noted that order parameters calculated from the dichroism of the solvent accessible $\nu_{\mathrm{a}}$ component give similar values for $\mathbf{E}$ when a significantly lower $\alpha$ of $20^{\circ}$ is assumed ( $c f$. ESI, $\dagger$ Fig. S13), while values with $\alpha>25^{\circ}$ did not yield physically meaningful values. The same calculation for $\mathbf{K}$ however yields lower order parameters for $\nu_{\mathrm{a}}$ compared to $\nu_{\mathrm{b}}$, while the basic potential dependent trends described above, are still found. This result may indicate that the absolute values for $\alpha$ change in the system, which is considered likely due to the significant structural changes that occur such as the KK dissociation and association.

The order parameters of EK calculated from $\nu_{\mathrm{b}}$ decrease during the negative scan and show constant values of $c a$. -0.3 at the hydrophobic interface and increase again during the positive scan (Table 1 and Fig. 7b). These results are more difficult to understand due to the number of possible involved states. As shown above both $\mathbf{E}$ and $\mathbf{K}$ adsorb in a parallel orientation as monomeric helices to the hydrophobic interface and $\mathbf{K}$ does this after dissociation from adsorbed KK. Because, in solution, EK is always in equilibrium with its monomers $\mathbf{E}$ and $\mathbf{K}$ all these states mentioned before as well as adsorbed EK may adsorb at the interface, which is indistinguishable by the amide $I^{\prime}$ band. Thus, a preferential adsorption of one form cannot be deduced from this data and most probably a mixture of the different possible forms is adsorbed. The observed biphasic adsorption during the negative scan also supports this view. The minimum order parameters and the halted adsorption on the hydrophobic interface can be understood similarly to $\mathbf{K}$ as reversible down tilt of some helices due to a hydrophobic interface binding. But different than in the case of $\mathbf{K}$, the surface concentration decreases on the hydrophilic interface during the positive scan. The remaining surface concentration may indicate a slow exchange process of desorbing $\mathbf{E}$ and adsorbing $\mathbf{K}$ because of the favored electrostatics of the positively charged $\mathbf{K}$ at the negatively charged interface.

\subsection{A sensitive balance between hydrophobic and electrostatic interactions governs the surface attachment}

The adsorption of $\mathbf{E}$ and $\mathbf{K}$ to the switchable germanium surface (Fig. 8) is, as well as protein adsorption to any static interface, governed by the protein's and the surface's charge and hydrophobicity. As was found for many other proteins, ${ }^{16,55}$ electrostatic interactions clearly dictate the adsorption behaviour on the hydrophilic surface and lead to the different behaviour of negatively charged $\mathbf{E}$ and positively charged K. Additionally, intramolecular interactions allow for a dense packing of $\mathbf{K}$ helices on the hydrophilic interface. However, on the hydrophobic surface $\mathbf{E}$ and a fraction of the $\mathbf{K}$ molecules adsorb in a similar fashion, i.e. as amphipathic helices with an almost flat orientation. Most proteins adsorb to electrically neutral hydrophobic surfaces, ${ }^{16,56}$ but it was argued that the adsorption on hydrophobic charged surfaces is difficult to predict. ${ }^{16}$ For $\mathbf{E}$ and
K apparently the amphipathic helical peptide structure ( $c f$. Fig. 1) is the common factor determining the similarities in the surface attachment. This propensity of the amphipathic helices to fold on hydrophobic interfaces opposes the behaviour of many globular proteins that tend to unfold on these interfaces. For $\mathbf{E}$ the unfavourable electrostatics, preventing adsorption on the hydrophilic interface, can be overcompensated by the interaction of the hydrophobic germanium surface with the hydrophobic face of the peptide helix. For $\mathbf{K}$ these hydrophobic interactions lead to reversible orientational reordering. In accordance with this observation, different binding conformations of the same protein on separated hydrophobic and hydrophilic surfaces have been reported for instance for amphipathic helical peptides experimentally, ${ }^{57-59}$ and theoretically. ${ }^{60,61}$ Also, a change of lysozyme orientation on switchable SAMs has been predicted theoretically. ${ }^{7}$ Here, the reorientation of peptides was observed in situ on a switchable hydrophobic/hydrophilic surface.

\section{Conclusion}

The hydrophobicity change induced by the reversible electrochemical termination switch of germanium surfaces - shown here for germanium (100) - in aqueous solutions can be employed as a 'smart' interface to reversibly trigger hydrophobic adsorption or adsorbate reorientation processes. The switching was realized at physiological pH in deuterated buffer which are prerequisites to employ the switching to study peptide and protein dynamics in situ by ATR-IR. Using in situ ATR-IR spectroscopy, the adsorption behaviour of the amphipathic coiled-coil forming peptides $\mathbf{E}$ and $\mathbf{K}$ was shown to be dictated by the surface charge and hydrophobicity. A reversible on/off switching of the hydrophobically driven adsorption of the negatively charged $\mathbf{E}$ was realized. Furthermore, a reversible orientation switching of adsorbed helical $\mathbf{K}$ was demonstrated. It is expected that this 'smart' germanium interface can be used to study adsorption dynamics of a wide variety of proteins and peptides, in situ.

\section{Materials and methods}

\subsection{Materials}

Deuterated phosphate buffer (d-PB) was prepared from $\mathrm{K}_{2} \mathrm{HPO}_{4}$ and $\mathrm{KH}_{2} \mathrm{PO}_{4}$ (VWR PROLABO Chemicals) in $\mathrm{D}_{2} \mathrm{O}$ (Merck, min. 99.9\% deuterium) at $\mathrm{pD}$ 7.4. The $\mathrm{pD}$-value was measured with a $\mathrm{pH}$ electrode that was immersed in $\mathrm{D}_{2} \mathrm{O}$ for $30 \mathrm{~min}$ and calculated by $\mathrm{pD}=\mathrm{pH}+0.4 .^{62}$ The $\mathrm{pD}$ was adjusted with a $20 \% \mathrm{DCl}$ solution (Merck, min. $99.5 \%$ deuterium) in $\mathrm{D}_{2} \mathrm{O}$.

The used coiled-coil peptides have the primary sequence: E: AcO-(EIAALEK) $)_{3} \mathrm{GW}-\mathrm{NH}_{2}$ and $\mathbf{K}$ : AcO-(KIAALKE) ${ }_{3} \mathrm{GW}-\mathrm{NH}_{2}$. The peptides were synthesized using standard Fmoc-chemistry on a CEM Liberty Blue microwave-assisted peptide synthesizer and purified by RP-high-performance liquid chromatography to yield a purity $>95 \%$. Identity of the peptides was determined by liquid chromatography-mass spectrometry. Peptide stock 
solutions of $\mathbf{E}$ and $\mathbf{K}$ were prepared, and the concentration was determined by UV absorbance of tryptophan at $280 \mathrm{~nm}$.

Germanium crystals were bought cut to size $52 \times 20 \times$ $0.5 \mathrm{~mm}^{3}$ with an angle of incidence of $45^{\circ}$ (Supplier: Crystal GmbH Berlin, type/doping: n/Sb, orientation: (100), thickness: $500 \mu \mathrm{m})$.

\subsection{Electrochemical in situ polarised ATR-IR spectroscopy}

Electrochemical experiments were performed using an Iviumstat potentiostat (Ivium Technologies). All potentials are referenced to SHE. First, CV was measured in the range $-1.3 \mathrm{~V}$ to $0.2 \mathrm{~V}$ with a scan rate of $10 \mathrm{mV} \mathrm{s}^{-1}$. The open circuit potential (OCP) was measured for $30 \mathrm{~min}$ and simultaneously IR spectra were recorded. Then, peptide solution was added (final concentration: $3.6 \mu \mathrm{mol} \mathrm{l^{-1 }}$ ) and mixed, followed by another OCP measurement for $30 \mathrm{~min}$. Finally, potential step experiments were started, the potential was decreased starting from $-0.19 \mathrm{~V}$ to $-1.19 \mathrm{~V}$ in steps of $0.2 \mathrm{~V}$ and subsequently increased with the same step size. Each potential step lasted $10 \mathrm{~min}$. For the IR measurements a Bio-Rad Excalibur spectrometer with a nitrogen-cooled MCT detector was used. An automated ZnSe wire grid polariser was applied (PIKE Technologies) that was programmed to allow alternating measurements in $\mathrm{p}^{-}$and s-polarisation. IR spectra were measured with a resolution of $4 \mathrm{~cm}^{-1}$, coadding 256 scans per spectrum. Measurement of a single spectrum took $83 \mathrm{~s}$. For the used germanium crystals the depth of penetration in the wavenumber region $1000 \mathrm{~cm}^{-1}$ to $4000 \mathrm{~cm}^{-1}$ varies between $640 \mathrm{~nm}$ to $160 \mathrm{~nm}$.

First, the measured single beam IR spectra were referenced against the spectra at OCP without peptide in solution. For water vapor correction, a water vapor spectrum was manually subtracted applying a factor to reduce water vapor contributions as much as possible. The resulting spectra were baseline corrected with a linear baseline in the amide I band region $1600 \mathrm{~cm}^{-1}$ to $1692 \mathrm{~cm}^{-1}$. The area of the baseline corrected peaks was calculated by numerical integration. The dichroic ratio $R$ was calculated by

$$
R=\frac{A_{\|}}{A_{\perp}}
$$

with $A_{\|}$and $A_{\perp}$ the integrated absorbance peak intensities in parallel and perpendicular polarisation, respectively. For the experiment a three-electrode setup was integrated in a multiple reflection ATR-IR setup, using the ATR-crystal as the working electrode. The cell contained a platinum sheet as the counter electrode and a $\mathrm{Ag} / \mathrm{AgCl}\left(3 \mathrm{~mol} \mathrm{l}^{-1} \mathrm{KCl}\right)$ (SUPER-Dri-Ref, World Precision Instruments) microreference electrode. Prior to measurements the cell was purged with $\mathrm{N}_{2}$. The cell volume was $13 \times 13 \times 5 \mathrm{~mm}^{3}$. During the OCP measurements and the potential step experiments, IR spectra were recorded. For each potential step, four s- and p-polarised spectra were measured, alternately.

Because of their orientation dependency, measured polarised integrated absorbances $\left(A_{\|}\right.$and $\left.A_{\perp}\right)$ are not directly proportional to the surface concentration of the respective analytes in the ATR-IR setup. However, a surface concentration proportional dimension $A_{\Gamma}$ can be calculated ${ }^{26,27}$ by

$$
A_{\Gamma}=A_{\perp}\left(2-\frac{E_{0 x}^{r 2}}{E_{0 z}^{r 2}{ }^{2}}\right)+A_{\|} \frac{E_{0 y}^{r 2}}{E_{0 z}^{r 2}}
$$

Here, $E_{0 x, y, z}^{r}$ stand for the electric field amplitudes at the crystal surface $(z=0)$ in $x, y$ and $z$ directions that can be calculated by Fresnel's equations. ${ }^{27}$ Eqn (3) assumes formation of thin films with thicknesses $d$ much smaller than the penetration depth $d_{\mathrm{p}}$. The decay of the electric field, normal to the crystal interface (z-direction) is

$$
E_{x, y, z}^{r}(z)=E_{0 x, y, z}^{r} \mathrm{e}^{-z / d_{\mathrm{p}}}
$$

with

$$
d_{\mathrm{p}}=\frac{1}{2 \pi \tilde{\nu} n_{1}\left(\sin ^{2} \Theta-n_{3,1^{2}}\right)^{1 / 2}}
$$

with $\tilde{\nu}$ the wavenumber in vacuum, $\Theta$ the incident angle, $n_{1}$ and $n_{3}$ the refractive indices of $\mathrm{Ge}$ and $\mathrm{D}_{2} \mathrm{O}$ and $n_{3,1}=n_{3} / n_{1}$. If an integrated molar absorption coefficient $\left(\int \varepsilon(\tilde{\nu}) \mathrm{d} \tilde{\nu}\right)$ can be deduced, the surface concentration $\Gamma$ can be calculated ${ }^{26}$ by

$$
\Gamma=\frac{A_{\Gamma} d}{3 N \nu d_{\mathrm{e} \perp}^{\text {iso }} \int \varepsilon(\tilde{\nu}) \mathrm{d} \tilde{\nu}}
$$

which becomes independent of the layer thickness $d$ for a thin film for which applies ${ }^{27}$

$$
d_{\mathrm{e} \perp}^{\text {iso }}=\frac{1}{\cos \Theta} n_{2,1} d E_{0 y}^{r 2}
$$

Here $d_{\mathrm{e} \perp}^{\text {iso }}$ denotes an "effective thickness" for an isotropic sample measured in s-polarisation, $N$ is the number of reflections in contact with analyte, $\nu$ is the number of absorbing moieties (peptide bonds) per molecule, $n_{2,1}=n_{2} / n_{1}$ and $n_{2}$ the refractive index of the peptide layer. The integrated molar absorption coefficient $\left(\int \varepsilon(\tilde{\nu}) \mathrm{d} \tilde{\nu}\right)$ per amino acid residue was deduced from transmission FTIR spectra published earlier. ${ }^{34}$ In case of adsorbing peptides forming a monolayer, $d$ will be smaller than $3 \mathrm{~nm}$ and it is assumed that multilayer formation will not exceed layer thicknesses of $20 \mathrm{~nm}$, which is likely because as found in the results ( $c f$. Section 2.2), measured surface concentrations remain well below double layer coverage. Thus, the thin film approximation: $E^{r}(z) / E_{0}^{r} \approx 1$, is valid with values between 0.99 and 0.95 , and thus eqn (3) and (6) are approximately valid in this thickness regime. The number of reflections in contact with the sample $N$ depends on the geometry of the used crystal and the used cell. ${ }^{28}$ For the employed setup with the in situ cell on top of the long side of the crystal $N$ was calculated by:

$$
N=\frac{l_{\mathrm{c}}}{2 l}\left(\frac{l}{h \tan \Theta}-\frac{1}{\tan ^{2} \Theta}+1\right)
$$

Here $l$ is the length of the crystal long face, $l_{\mathrm{c}}$ is the length of the in situ cell in the direction of beam propagation and $h$ the crystal height. 
The order parameter of alpha helical peptides adsorbed on the germanium surface was calculated by ${ }^{53}$

$$
S=\frac{2\left(E_{0 x}^{r 2}-R E_{0 y}^{r 2}+E_{0 z}^{r 2}\right)}{\left(3 \cos ^{2} \alpha-1\right)\left(E_{0 x}^{r}{ }^{2}-R E_{0 y}^{r}{ }^{2}-2 E_{0 z}^{r}{ }^{2}\right)}
$$

where $\alpha$ stands for the angle between the molecular axis and the transition dipole moment.

For axial symmetric distribution of the helix axis around the interface normal with the tilt angle $\theta$ the order parameter is defined as:

$$
S=\frac{3\left\langle\cos ^{2} \theta\right\rangle-1}{2}
$$

The complete set of raw and processed data are available in a data package associated with this work. ${ }^{63}$

\section{Author contributions}

Conceptualisation (MR), formal analysis (LMB, MR), funding acquisition (MR), investigation (LMB, ALB, AE, MR), supervision ( $\mathrm{AE}, \mathrm{MR}$ ), writing of the original draft (LMB, MR), reviewing and editing (LMB, ALB, AE, MR).

\section{Conflicts of interest}

There are no conflicts of interest to declare.

\section{Acknowledgements}

This work was funded by the Deutsche Forschungsgemeinschaft (DFG, German Research Foundation) under Germany's Excellence Strategy - EXC 2033 - 390677874 - RESOLV. MR acknowledges funding from the European Unions Horizon 2020 research and innovation programme through a Marie Skłodowska-Curie Grant (Agreement No. 705857). Petra Ebbinghaus and Fang Niu are acknowledged for technical assistance. The MPIE workshop is acknowledged for design and manufacturing of the in situ cells. Open Access funding provided by the Max Planck Society.

\section{References}

1 Y. Liu, L. Mu, B. Liu, S. Zhang, P. Yang and J. Kong, Chem. Commun., 2004, 1194-1195.

2 L. Mu, Y. Liu, S. Zhang, B. Liu and J. Kong, New J. Chem., 2005, 29, 847-852.

3 L. Mu, Y. Liu, S. Cai and J. Kong, Chem. - Eur. J., 2007, 13, 5113-5120.

4 C. L. Yeung, P. Iqbal, M. Allan, M. Lashkor, J. A. Preece and P. M. Mendes, Adv. Funct. Mater., 2010, 20, 2657-2663.

5 H. Zeng, Y. Zhang, H. Nakajima and K. Uchiyama, Sens. Actuators, B, 2017, 251, 334-338.

6 X. Ma, A. Gosai, G. Balasubramanian and P. Shrotriya, J. Mater. Chem. B, 2017, 5, 3675-3685.
7 Y. Xie, W. Gong, J. Jin, Z. Zhao, Z. Li and J. Zhou, Appl. Surf. Sci., 2020, 506, 144962.

8 Y. Guan, D. Yu, H. Sun, J. Ren and X. Qu, Chem. Commun., 2021, 57, 2641-2644.

9 H. Nandivada, A. M. Ross and J. Lahann, Prog. Polym. Sci., 2010, 35, 141-154.

10 P. M. Mendes, Chem. Soc. Rev., 2008, 37, 2512-2529.

11 G. Lux, A. Langer, M. Pschenitza, X. Karsunke, R. Strasser, R. Niessner, D. Knopp and R. Rant, Anal. Chem., 2015, 87, 4538-4545.

12 A. Langer, P. A. Hampel, W. Kaiser, J. Knezevic, T. Welte, V. Villa, M. Maruyama, M. Svejda, S. Jähner, F. Fischer, R. Strasser and U. Rant, Nat. Commun., 2013, 4, 2099.

13 B. D. Kieviet, P. M. Schoen and G. J. Vansco, Lab Chip, 2014, 14, 4159-4170.

14 P. A. Fritz, R. M. Boom and C. Schroën, Sep. Purif. Technol., 2021, 118754.

15 M. Rabe, D. Verdes and S. Seeger, Adv. Colloid Interface Sci., 2011, 162, 87-106.

16 S. J. Attwood, R. Kershaw, S. Uddin, S. M. Bishop and M. E. Welland, J. Mater. Chem. B, 2019, 7, 2349-2361.

17 J. Lahann, S. Mitragotri, T.-N. Tran, H. Kaido, J. Sundaram, I. S. Choi, S. Hoffer, G. A. Somorjai and R. Langer, Science, 2003, 299, 371-373.

18 H. Gerischer and W. Mindt, Surf. Sci., 1966, 4, 440-451.

19 R. Memming and G. Neumann, J. Electroanal. Chem. Interfacial Electrochem., 1969, 21, 295-305.

20 F. Maroun, F. Ozanam and J.-N. Chazalviel, J. Phys. Chem. B, 1999, 103, 5280-5288.

21 F. Maroun, F. Ozanam and J.-N. Chazalviel, Surf. Sci., 1999, 427-428, 184-189.

22 J.-N. Chazalviel, A. Belaïdi, M. Safi, F. Maroun, B. Erné and F. Ozanam, Electrochim. Acta, 2000, 45, 3205-3211.

23 S. Nayak and A. Erbe, Phys. Chem. Chem. Phys., 2016, 18, 25100-25109.

24 F. Niu, R. Schulz, A. C. Medina, R. Schmid and A. Erbe, Phys. Chem. Chem. Phys., 2017, 19, 13585-13595.

25 F. Niu, M. Rabe, S. Nayak and A. Erbe, J. Chem. Phys., 2018, 148, 222824.

26 P. Wenzl, M. Fringeli, J. Goette and U. P. Fringeli, Langmuir, 1994, 10, 4253-4264.

27 U. P. Fringeli, In Situ Infrared Attenuated Total Reflection Membrane Spectroscopy, in Internal reflection spectroscopy: theory and applications, ed. F. Mirabella, Marcel Dekker, 1993, pp. 255-324.

28 N. J. Harrick, Internal reflection spectroscopy, Harrick Scientific Corp, Ossining, NY, 1987.

29 C. F. Carter, H. Lange, S. V. Ley, I. R. Baxendale, B. Wittkamp, J. G. Goode and N. L. Gaunt, Org. Process Res. Dev., 2010, 14, 393-404.

30 J. Greener, B. Abbasi and E. Kumacheva, Lab Chip, 2010, 10, 1561-1566.

31 K. K. Sriram, S. Nayak, S. Pengel, C.-F. Chou and A. Erbe, Analyst, 2017, 142, 273-278.

32 J. R. Litowski and R. S. Hodges, J. Biol. Chem., 2002, 277, 37272-37279. 
33 M. Rabe, C. Schwieger, H. R. Zope, F. Versluis and A. Kros, Langmuir, 2014, 30, 7724-7735.

34 M. Rabe, H. R. Zope and A. Kros, Langmuir, 2015, 31, 9953-9964.

35 M. Rabe, C. Aisenbrey, K. Pluhackova, V. de Wert, A. L. Boyle, D. F. Bruggeman, S. A. Kirsch, R. A. Böckmann, A. Kros, J. Raap and B. Bechinger, Biophys. J., 2016, 111, 2162-2175.

36 J. P. Segrest, H. D. Loof, J. G. Dohlman, C. G. Brouillette and G. M. Anantharamaiah, Proteins: Struct., Funct., Genet., 1990, 8, 103-117.

37 V. K. Mishra and M. N. Palgunachari, Biochemistry, 1996, 35, 11210-11220.

38 G. Grigoryan and A. Keating, Curr. Opin. Struct. Biol., 2008, 18, 477-483.

39 S. Rivillon, Y. J. Chabal, F. Amy and A. Kahn, Appl. Phys. Lett., 2005, 87, 253101.

40 R. W. Hendler and R. I. Shrager, J. Biochem. Biophys. Methods, 1994, 28, 1-33.

41 M. Rabe, J. Open Res. Software, 2020, 8, 13.

42 J.-J. Max and C. Chapados, J. Chem. Phys., 2002, 116, 4626-4642. 43 Y. Chabal, Surf. Sci., 1986, 168, 594-608.

44 J. E. Crowell and G. Lu, J. Electron Spectrosc. Relat. Phenom., 1990, 54-55, 1045-1057.

45 A. Barth, Biochim. Biophys. Acta., 2007, 1767, 1073-1101.

46 M. Rabe, A. Boyle, H. R. Zope, F. Versluis and A. Kros, J. Pept. Sci., 2014, 104, 65-72.

47 D. Lin-Vien, N. B. Colthup, W. G. Fateley and J. G. Grasselli, The Handbook of Infrared and Raman Characteristic Frequencies of Organic Molecules, Elsevier, 1991, pp. 117-154.

48 S. T. R. Walsh, R. P. Cheng, W. W. Wright, D. O. V. Alonso, V. Daggett, J. M. Vanderkooi and W. F. DeGrado, Protein Sci., 2003, 12, 520-531.
49 A. Barth and C. Zscherp, Q. Rev. Biophys., 2002, 35, 369-430. 50 E. S. Manas, Z. Getahun, W. W. Wright, W. F. DeGrado and J. M. Vanderkooi, J. Am. Chem. Soc., 2000, 122, 9883-9890.

51 T. Blundell, D. Barlow, N. Borkakoti and J. Thornton, Nature, 1983, 306, 281-283.

52 E. Goormaghtigh, V. Raussens and J.-M. Ruysschaert, Biochim. Biophys. Acta, 1999, 1422, 105-185.

53 L. K. Tamm and S. A. Tatulian, Q. Rev. Biophys., 1997, 30, 365-429.

54 D. Marsh, M. Müller and F.-J. Schmitt, Biophys. J., 2000, 78, 2499-2510.

55 W. Norde, Colloids Surf., B, 2008, 61, 1-9.

56 J. D. Andrade and V. Hlady, Biopolymers/Non-Exclusion HPLC, Springer Berlin Heidelberg, 1986, pp. 1-63.

57 S. Noinville, F. Bruston, C. E. Amri, D. Baron and P. Nicolas, Biophys. J., 2003, 85, 1196-1206.

58 O. Mermut, D. C. Phillips, R. L. York, K. R. McCrea, R. S. Ward and G. A. Somorjai, J. Am. Chem. Soc., 2006, 128, 3598-3607.

59 D. C. Phillips, R. L. York, O. Mermut, K. R. McCrea, R. S. Ward and G. A. Somorjai, J. Phys. Chem. C, 2007, 111, 255-261.

60 S. Roy, T. L. Naka and D. K. Hore, J. Phys. Chem. C, 2013, 117, 24955-24966.

61 K. D. Krause, S. Roy and D. K. Hore, Biointerphases, 2017, 12, $02 \mathrm{D} 407$.

62 P. K. Glasoe and F. A. Long, J. Phys. Chem., 1960, 64, 188-190.

63 L.-M. Baumgartner, A. Erbe, A. L. Boyle and M. Rabe, Data package: Controlling Amphipathic Peptide Adsorption by Smart Switchable Germanium Interfaces, EDMOND, Max Planck Digital Library. 